\title{
EDITORIAL \\ Long-term solutions in neurosurgery using extended reality technologies
}

\author{
*Gagandeep Singh, MD, ${ }^{1}$ Tejasvi Kainth, MD, ${ }^{2}$ Nihal Manjila, ${ }^{3}$ Shubham Jain, PhD, ${ }^{4}$ \\ Anatoliy Vaysberg, MD, ${ }^{1}$ Vadim Spektor, MD, ${ }^{5}$ Prateek Prasanna, PhD, ${ }^{2}$ and Sunil Manjila, MD ${ }^{6}$ \\ ${ }^{1}$ Neuroradiology Division, Department of Radiology, Newark Beth Israel Medical Center, Newark, New Jersey; Departments \\ of ${ }^{2}$ Biomedical Informatics and ${ }^{4}$ Computer Science, Stony Brook University, Stony Brook; and ${ }^{5}$ Neuroradiology Division, \\ Department of Radiology, Columbia University Medical Center, New York, New York; ${ }^{3}$ Department of Biology, Case Western \\ Reserve University, Cleveland, Ohio; and ${ }^{6}$ Department of Neurosurgery, Mountain View Regional Medical Center, Las Cruces, \\ New Mexico
}

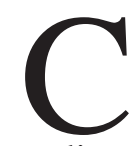

LINICAL practice in neurosurgery has now adapted to support enhanced digital visualizations for training and surgical planning using mixed reality (MR), virtual reality (VR), and augmented reality (AR). These extended reality technologies have been safely used to explore the operative field from different viewpoints, visualizing the neurovascular anatomy hidden from the surgical field, thereby offering an enhanced comprehensive sensory experience, especially in keyhole approaches to deep-lying targets., ${ }^{1,2}$ Together with the fusion of additional imaging data such as indocyanine green (ICG), 5-aminolevulinic acid (5-ALA), or fluorescein angiography, MR has increased the precision in neurosurgical procedures. With work hour limitations and erstwhile COVID-19 restrictions, these technological advances also offer exemplary training and practicing tools for both novices and experts alike, resulting in their recent exponential growth. In addition, MR can be utilized for telecasting, patient education, and long-distance telecollaboration, and it can help bridge the global educational gap in the field of neurosurgery, including the scope of credentialing and recertifications..$^{3-6}$

\section{Virtual Reality}

Based on the level of immersion, VR can be classified into non-immersive, semi-immersive, and fully immersive VR. With non-immersive VR, the virtual environment is viewed through a window on a standard monitor. Keyboard, mouse, or enhanced 3D interaction devices are used to interact with non-immersive VR. Semi-immersive VR combines high-performing graphics with a large screen projector, or multiple display projections, to widen the field of view and provide the user with an enhanced immersive user experience. Fully immersive VR provides the most direct and complete interactive experience with a virtual world using a head-mounted display device. Most types of surgical VR are immersive experiences that allow the operator to perform exact surgical steps as done with a patient, hence providing a recall-based (déjà vu) training experience.

Virtual intracranial visualization and navigation (VIVIAN) was a preoperative neurosurgical planning system developed in the 1990s for the Dextroscope, a VR surgical planning platform. Precision VR (Surgical Theater, Inc.) has been used in skull base tumors both in open craniotomies and in expanded endoscopic endonasal methods. Preoperative planning using VR has been shown to evaluate the dimensions of the surgical corridors and identify their boundaries and relevant neurovascular anatomy. ${ }^{7}$ With these numerous examples in the literature, evidence seems to support that VR can improve the efficacy and safety of neurosurgical procedures..$^{7-9}$ For spinal procedures, VR training technology can improve physician comfort with technical skills as well as spatial awareness in procedures such as pedicle screw insertions and vertebroplasty. ${ }^{9}$

Lee and Wong, ${ }^{8}$ while examining the role of VR in tumor management, found that the technology was beneficial in surgical planning, navigation, and training. VR interfaces may also include haptic feedback, as in the case of CyberGrasp (Immersion Corp., https://www.immersion. com) and Phantom (https://www.3dsystems.com/haptics), which provide tactile and kinesthetic responses to users. Cohen et al..$^{10}$ published a study on the utility of haptic interface and VR creation of endoscopic third ventriculostomy in 2013, citing the relevance of VR in training

${ }^{*}$ P.P. and S.M. share senior authorship of this work. 
neuroendoscopy. Haptic feedback is a necessary addition to VR training systems to maximize skill transferability between training scenarios and a clinical setting. ${ }^{11}$ Zeiger et al. ${ }^{12}$ published their findings on an MR platform, offering intraoperative navigation using $3 \mathrm{D}$ reconstructions of patient anatomy for endoscopic endonasal skull base surgery. MR has significantly improved education and training among neurosurgery residents. Hooten et al. ${ }^{13}$ evaluated 260 residents who participated in a stereotactic simulation in which the virtual projection of a physical catheter onto a virtual model of ventricles was used for familiarization with the ventriculostomy procedure.

\section{Augmented Reality}

During surgery, anatomical structures segmented in VR can be projected into the oculars of a navigationtracked microscope as AR objects, which can provide the surgeon with 3D spatial relationships including depth perception. ${ }^{14}$ Cranial neurosurgery has utilized AR to localize and excise gliomas, meningiomas, arteriovenous malformations, and clip aneurysms. ${ }^{1}$ Umebayashi et al. ${ }^{2}$ used $\mathrm{O}$-arm (Medtronic) intraoperative imaging to create an augmented image in a navigation system connected with a microscope that combined AR with the real view. AR helped with the surgical field orientation and enabled surgeons to navigate the electric drill using a navigation map track. Mascitelli et al. ${ }^{1}$ retrospectively studied 79 patients with 85 intracranial pathologies and identified intracranial lesions, specific nerves, and vessels with excellent accuracy in $71.4 \%$ of lesions by using a navigation-linked heads-up display. Cabrilo et al. ${ }^{15}$ prospectively studied 28 patients with vascular aneurysms who underwent aneurysm clipping using a computer-generated $3 \mathrm{D}$ rendering of the patient's anatomy projected into the eyepiece of an operating microscope. They reported a neuronavigation coregisteration accuracy of $100 \%$ and AR-assisted accurate clip placement in $92.3 \%$ of cases. Jean ${ }^{16}$ used a 3D VR rendering of a clinoid meningioma using the Surgical Navigation Advanced Platform (Surgical Theater Inc.) for preoperative rehearsal of an approach for mini-pterional craniotomy with extradural clinoidectomy. During the rehearsal, the optimal opening was saved as a VR file and was projected intraoperatively into the eyepiece of a navigation-tracked microscope (SyncAR version 3.8.0, Surgical Theater, Inc.). Using AR, the planned surgical opening was then visible as an overlay on the patient in real time during the surgery. Weeks et al. ${ }^{3}$ demonstrated the use of AR-based immersive learning for improving short-term recall compared with conventional 2D screenbased review, which potentially increases engagement of millennial learners. In a study by Yudkowsky et al., ${ }^{4} 16$ neurosurgery residents showed improvement in successful first-pass cannulations after practicing a simulation-based ventriculostomy procedure using the ImmersiveTouch system. AR has been successfully used to broadcast and demonstrate live procedures in Paraguay and Brazil..$^{5}$ Virtual interactive presence and AR (VIPAR) is an iPad-based tool that uses the internet to provide virtual assistance. ${ }^{6}$ This allows experienced surgeons to provide guidance remotely, promotes long-distance intraoperative telecollaboration, and enhances global neurosurgical education.

\section{Current Limitations}

There are many limitations for using AR devices in surgery. If head-mounted displays are used, they need to be light, mobile, comfortable to wear, and functional for long hours. ${ }^{17}$ Current AR/VR technology has limitations such as large appliances, cables, and a limited battery life. Limited visualization of deeper brain lesions due to altered depth of perception via AR could potentially confuse a surgeon's sensory modalities. ${ }^{18}$ Intraoperative brain shift or cisternal vasculature secondary to CSF loss during surgery may provide an inaccurate image to a surgeon using AR/VR models. VR often poses a steep learning curve, thereby limiting neurosurgeons from transferring their skills preoperatively into practice in a real patient scenario. ${ }^{19}$ Lack of haptic feedback and adaptive tissue response such as vascularity, firmness, and tissue texture in VR training platforms are additional challenges faced during preoperative planning and rehearsal. ${ }^{7}$ Data management and patient confidentiality pose another set of hurdles, as witnessed by the application of electronic medical records in healthcare. ${ }^{20}$ It is imperative that clinicians and their supporting staff maintain the legal and ethical obligations to anonymized storage, review, or transfer of data with the utmost sensitivity. Encryption software can enhance confidentiality and confidence but does not guarantee prevention of data hijacking, which leaves us vulnerable to increased medical identity thefts. ${ }^{21}$ Another significant challenge for the translation of VR systems into clinical workflow stems from a regulatory perspective. VR systems used in medical treatment are overseen and regulated by the FDA. Specifically, the FDA classifies VR systems as novel digital health technologies alongside innovations such as implanted and wearable wireless devices. Manufacturers and distributors of VR systems have to abide by the FDA regulations for software in a medical device (SiMD) or software as a medical device (SaMD) depending on what form the VR system takes (https://www.fda.gov/regulatory-information/search-fdaguidance-documents/policy-device-software-functionsand-mobile-medical-applications).

\section{Broader Applications}

Integration of extended reality into other domains of technology has led to major disruptions. AR/VR integration with robotics could help provide feedback through tactile, auditory, and augmented visual loop cueing, thus decreasing the workload and the learning curve for the user. ${ }^{22}$ Going a step further, the robotics and AR model could then be modulated to function remotely using verbal commands or sensor-control devices like gloves that can capture user hand movements and provide a sensory feedback loop to the user. ${ }^{23}$ Emerging techniques such as radiomics (subvisual characterization of radiological imaging) can also be used in conjunction with VR to provide complementary information regarding tissue morphology and texture. ${ }^{24}$ Currently, MR has been incorporated into selected neurosurgical research labs in the United States. ${ }^{25}$ With the advent of practical and user-friendly devices coupled with high-speed internet, artificial intelligence (AI), and robotics, extended reality technology has the potential to effectively train surgeons worldwide. And it can sub- 
stantially improve patient experience and postoperative clinical outcomes.

In a wider arena, MR has been helpful in allied neuroscience areas especially for spatial navigation, memory tasks like calculation, and by providing visual cues like directional arrows and landmarks in patients with Alzheimer's disease. ${ }^{26}$ Additionally, platforms like the VR exercise app for dementia and Alzheimer's (VRADA) have significantly high acceptability, usability, and tolerability among varied age groups. ${ }^{27} \mathrm{We}$ can use extended realities to imitate complicated walking environments for movement training and assessment while maintaining a safe and well-protected environment for patients poststroke, those with Parkinson's disease, and others with movement disorders. ${ }^{28}$ Avenues are open to explore neurorehabilitation combined with physical rehabilitation as an application of this technology. In 2020, with the approval of the first prescription-only game-based digital therapeutic device in children with attention deficit hyperactivity disorder, it is evident that we are entering into the digital therapeutic era. ${ }^{29}$

\section{The Future of MR in Neurosurgery}

Traditional head-mounted displays have given way to nuances like HoloLens smart glasses and glass-mounted displays in operating rooms; real-time display of diffusion tensor (high-density fiber tracking) imaging during surgery can also be utilized in AR to avoid inadvertent transection of pyramidal fibers during intraaxial tumor resection. Emerging technologies such as Quicktome and Infinitome (Omniscient Neurotechnology Pty.) that are based on the Human Connectome Project (HCP; https:// www.humanconnectome.org) can be integrated onto these AR/VR platforms. Currently, the former is only a $2 \mathrm{D}$ visualization software showing the enormous possibilities of parcellations and novel network areas in the brain of varying eloquence, developed based on $\mathrm{AI}$ in $\mathrm{HCP}$ data. However, this can be easily integrated with a 3D planning and practicing software for tumor resections.

With the advent of Visualase (Medtronic) and robotic autoguide treatments of deep intracranial lesions, as well as AR/VR-based stereoelectroencephalography-led placement, the new era of AR/VR in neurosurgery looks exciting and promising. Incorporation of adjunct technologies such as Quicktome and robotics, along with fusion of imaging modalities like ICG and 5-ALA, will change the landscape further by increasing the accuracy of surgical interventions. In the face of a global pandemic, we foresee increased incorporation of AR/VR into our existing clinical workflows to improve learning efficiency and the safety of neurosurgical procedures. The field of neurosurgery has pioneered the use of such techniques in clinical procedures; these technologies continue to rapidly evolve with advances in computing power and development of novel machine learning techniques. Extended reality tools will continue to profoundly impact the neurosurgery landscape, drive technology investment, and lead to better clinical care with reduced morbidities.

https://thejns.org/doi/abs/10.3171/2021.5.FOCUS21235

\section{Acknowledgments}

We appreciate the insightful academic input and in-depth final edits of the topic editor, Dr. Walter Jean.

\section{References}

1. Mascitelli JR, Schlachter L, Chartrain AG, Oemke H, Gilligan J, et al. Navigation-linked heads-up display in intracranial surgery: early experience. Oper Neurosurg (Hagerstown). 2018;15(2):184-193.

2. Umebayashi D, Yamamoto Y, Nakajima Y, Fukaya N, Hara $\mathrm{M}$. Augmented reality visualization-guided microscopic spine surgery: transvertebral anterior cervical foraminotomy and posterior foraminotomy. J Am Acad Orthop Surg Glob Res Rev. 2018;2(4):e008.

3. Weeks JK, Pakpoor J, Park BJ, Robinson NJ, Rubinstein NA, et al. Harnessing augmented reality and CT to teach firstyear medical students head and neck anatomy. Acad Radiol. 2020;28(6):871-876.

4. Yudkowsky R, Luciano C, Banerjee P, Schwartz A, Alaraj $\mathrm{A}$, et al. Practice on an augmented reality/haptic simulator and library of virtual brains improves residents' ability to perform a ventriculostomy. Simul Healthc. 2013;8(1):25-31.

5. Datta N, MacQueen IT, Schroeder AD, Wilson JJ, Espinoza JC, et al. Wearable technology for global surgical teleproctoring. J Surg Educ. 2015;72(6):1290-1295.

6. Davis MC, Can DD, Pindrik J, Rocque BG, Johnston JM. Virtual interactive presence in global surgical education: international collaboration through augmented reality. World Neurosurg. 2016;86:103-111.

7. Jean WC, Singh A. Expanded endoscopic endonasal transtuberculum approach for tuberculum sellae meningioma: operative video with 360-degree fly-through and surgical rehearsal in virtual reality: 2-dimensional operative video. Oper Neurosurg (Hagerstown). 2020;19(2):E179-E180.

8. Lee C, Wong GKC. Virtual reality and augmented reality in the management of intracranial tumors: a review. J Clin Neurosci. 2019;62:14-20.

9. Fiani B, De Stefano F, Kondilis A, Covarrubias C, Reier L, Sarhadi K. Virtual reality in neurosurgery: "can you see it?"-a review of the current applications and future potential. World Neurosurg. 2020;141:291-298.

10. Cohen AR, Lohani S, Manjila S, Natsupakpong S, Brown N, Cavusoglu MC. Virtual reality simulation: basic concepts and use in endoscopic neurosurgery training. Childs Nerv Syst. 2013;29(8):1235-1244.

11. Bernardo A. Virtual reality and simulation in neurosurgical training. World Neurosurg. 2017;106:1015-1029.

12. Zeiger J, Costa A, Bederson J, Shrivastava RK, Iloreta AMC. Use of mixed reality visualization in endoscopic endonasal skull base surgery. Oper Neurosurg (Hagerstown). 2020;19(1):43-52.

13. Hooten KG, Lister JR, Lombard G, Lizdas DE, Lampotang $\mathrm{S}$, et al. Mixed reality ventriculostomy simulation: experience in neurosurgical residency. Neurosurgery. 2014;10(suppl 4):576-581.

14. Guha D, Alotaibi NM, Nguyen N, Gupta S, McFaul C, Yang VXD. Augmented reality in neurosurgery: a review of current concepts and emerging applications. Can J Neurol Sci. 2017;44(3):235-245.

15. Cabrilo I, Bijlenga P, Schaller K. Augmented reality in the surgery of cerebral aneurysms: a technical report. Neurosurgery. 2014;10(suppl 2):252-261.

16. Jean WC. Mini-pterional craniotomy and extradural clinoidectomy for clinoid meningioma: optimization of exposure using augmented reality template: 2-dimensional operative video. Oper Neurosurg (Hagerstown). 2020;19(6):E610.

17. Khor WS, Baker B, Amin K, Chan A, Patel K, Wong J. Augmented and virtual reality in surgery-the digital surgical 
environment: applications, limitations and legal pitfalls. Ann Transl Med. 2016;4(23):454.

18. Elmi-Terander A, Skulason H, Söderman M, Racadio J, Homan R, et al. Surgical navigation technology based on augmented reality and integrated 3D intraoperative imaging: a spine cadaveric feasibility and accuracy study. Spine (Phila $\mathrm{Pa}$ 1976). 2016;41(21):E1303-E1311.

19. Zhalmukhamedov E, Urakov TM. Current and future use of virtual and augmented reality in neurosurgery: a literature review. Indones J Neurosurg. 2019;2(2):50-54.

20. Haynes CL, Cook GA, Jones MA. Legal and ethical considerations in processing patient-identifiable data without patient consent: lessons learnt from developing a disease register. J Med Ethics. 2007;33(5):302-307.

21. Mancilla D, Moczygemba J. Exploring medical identity theft. Perspect Health Inf Manag. 2009;6(Fall):1e.

22. L'Orsa R, Macnab CJ, Tavakoli M. Introduction to haptics for neurosurgeons. Neurosurgery. 2013;72(suppl 1):139-153.

23. Madhavan K, Kolcun JPG, Chieng LO, Wang MY. Augmented-reality integrated robotics in neurosurgery: are we there yet? Neurosurg Focus. 2017;42(5):E3.

24. Singh G, Manjila S, Sakla N, True A, Wardeh AH, et al. Radiomics and radiogenomics in gliomas: a contemporary update. Br J Cancer. Published online May 6, 2021. doi:10.1038/s41416-021-01387-w

25. Lessick S, Kraft M. Facing reality: the growth of virtual reality and health sciences libraries. J Med Libr Assoc. 2017;105(4):407-417.

26. Cogné M, Auriacombe S, Vasa L, Tison F, Klinger E, et al. Are visual cues helpful for virtual spatial navigation and spatial memory in patients with mild cognitive impairment or Alzheimer's disease? Neuropsychology. 2018;32(4):385-400.
27. Hassandra M, Galanis E, Hatzigeorgiadis A, Goudas M, Mouzakidis C, et al. A virtual reality app for physical and cognitive training of older people with mild cognitive impairment: mixed methods feasibility study. JMIR Serious Games. 2021;9(1):e24170.

28. Asakawa T, Sugiyama K, Nozaki T, Sameshima T, Kobayashi $\mathrm{S}$, et al. Can the latest computerized technologies revolutionize conventional assessment tools and therapies for a neurological disease? The example of Parkinson's disease. Neurol Med Chir (Tokyo). 2019;59(3):69-78.

29. FDA Permits Marketing of First Game-Based Digital Therapeutic to Improve Attention Function in Children with ADHD. US Food and Drug Administration; 2020. Accessed June 7, 2021. https://www.fda.gov/news-events/pressannouncements/fda-permits-marketing-first-game-baseddigital-therapeutic-improve-attention-function-children-adhd

\section{Disclosures}

The authors report no conflict of interest.

\section{Correspondence}

Gagandeep Singh: gagan32092@gmail.com.

\section{INCLUDE WHEN CITING}

DOI: 10.3171/2021.5.FOCUS21235. 